\title{
Economic Evaluation of Vessel Traffic Service (VTS) Based on Survey Data and I.O. Analysis
}

\author{
Zeng Luling ${ }^{1,2}$, Jiang Zhengxiong ${ }^{1}$, Ren Lvzhen ${ }^{2, ~ *, ~ D u a n ~ X i n g f e n g ~}{ }^{2}$ \\ ${ }^{1}$ Law School, Shanghai Maritime University, Shanghai, China \\ ${ }^{2}$ Navigation College, Jimei University, Xiamen, China \\ Email address: \\ zeng@jmu.edu.cn (Zeng Luling), zxjiang@shmtu.edu.cn (Jiang Zhengxiong), lzren@jmu.edu.cn (Ren Lvzhen), \\ dxf-yk@163.com (Duan Xingfeng) \\ *Corresponding author
}

\section{To cite this article:}

Zeng Luling, Jiang Zhengxiong, Ren Lvzhen, Duan Xingfeng. Economic Evaluation of Vessel Traffic Service (VTS) Based on Survey Data and I.O. Analysis. American Journal of Traffic and Transportation Engineering. Vol. 6, No. 5, 2021, pp. 139-146.

doi: $10.11648 /$ j.ajtte.20210605.12

Received: September 22, 2021; Accepted: October 20, 2021; Published: October 29, 2021

\begin{abstract}
The functions of VTS are to ensure the security of waterways in the port, improve the efficiency of vessel navigation and avoid the pollution of the water, which is an important approach to water traffic management. It has apparent achievements in enhancing port operation efficiency and promoting the development of related industries. As a public service, the investment and operation cost of VTS is high. It has significant indirect economic and social benefits but relatively small direct economic benefits or profits. There is a lack of a unified method for the quantitative assessment of the VTS economic benefits. It is a meaningful topic that should be further explored in theoretical research. This paper analyzes and evaluates the connotation of VTS economic benefits and defines the scope of direct and indirect economic benefits. The direct economic benefits are classified with the specific business of VTS to establish a basic model, and sampling analysis is used to measure them in the application. For indirect economic benefits, the Input-Output Analysis (I-O) is used to measure them. The operational data of the Tianjin VTS is used as an example for verification. The correct understanding and evaluation of direct and indirect economic benefits generated by VTS is an inherent requirement to reflect the operational performance of VTS objectively and provides a scientific decision-making basis for the continuous investment and operation of VTS.
\end{abstract}

Keywords: Vessel Traffic Service, Economic Evaluation, Economic Benefits, Input-output Analysis

\section{Introduction}

Vessel Traffic Services (VTS) is a system established by port administration to implement traffic control and provide consulting services to vessels, ensure the security of waterways, improve traffic efficiency, and protect the water environment. The system consists of radar, AIS (Automatic Identification System), VHF (Very High Frequency), network and transmission, multi-sensor processing, hydro-meteorology, information display and data processing subsystem. It collects and processes information to provide traffic surveillance, information service, navigation advisory, traffic organization, and supports joint operations. It improves channel usage efficiency and reduces the risk of traffic accidents and environmental pollution by dynamically monitoring and organizing vessels traffic flow. So, the VTS is an essential active traffic management technology in port management.

As a public service, the investment and operation of VTS are characterized by enormous investment costs, a long construction period and high operation and maintenance costs. It has significant indirect economic and social benefits but relatively small direct economic benefits or profits. Therefore, the correct understanding and assessment of the economic benefits generated by the operation of VTS are meaningful to objectively reflect the operational performance of VTS and provide a scientific decision-making basis for the continuous investment and operation of VTS.

After decades of development of the VTS system, besides system function, planning and operation evaluation, related research has been extended to evaluate its operation's economic and social benefits. Hans (1976) made an early 
scientific classification and systematic study on the cost-effectiveness of VTS and proposed a qualitative evaluation and analysis approach [1]. Fang et al. (1990) quantified the economic benefits of VTS using a data sampling and weighted average algorithm. This idea and model were adopted and specified by Tian (2000) [2], Shao et al. (2000) [3], Liu et al. (2001) [4] and applied in the evaluation of different port VTS. George Bushell et al. (1992) used quantitative methods to analyze and calculate the economic benefits of VTS in terms of hazard reduction and environmental protection [5]. The International Association of Marine Aids to Navigation and Lighthouse Authorities (IALA) presented a complete Cost-Benefit Analysis (CBA) framework for VTS in the 2008 edition of the VTS manual. The benefit analysis (CBA) framework in the 2016 edition of the VTS manual provided a brief description of the analysis and calculation of VTS cost-benefit [6]. Xie (2010) used the Delphi method and hierarchical analysis for quantitative estimation [7]. Lu (2016) employs the Cost-Benefit Analysis and Fuzzy Comprehensive Evaluation method to testify Qingdao VTS is a practical public project [8]. Li (2010) [9], Cao (2018) 10] synthesized the development results of VTS economic benefits assessment and quantified the financial contribution of VTS in Guangzhou. Lee, G et al.(2015) used the contingent valuation survey to elicit their willingness to pay for VTS to determining the economic feasibility of VTS [11]. Jun-Min Mou et al.(2015) set up an evaluation with four key indicators (safety, traffic efficiency, environmental protection and reducing supervising cost) and the 10-year data of Zhoushan Port, which was illuminating [12].

From the early qualitative evaluation to the quantitative evaluation of direct economic benefits, and then to the quantitative evaluation of indirect and social benefits that have been explored in recent years, the theory and technology of VTS benefit evaluation have been continuously improved. However, extensive and detailed investigation and data analysis with specific cases are needed to acquire and analyze operational data and determine critical parameters. This research modifies the methods mentioned in the above literature, classifies the types of direct economic benefits based on the rich operational data of Tianjin port, forms the calculation formula, and carries out the indirect benefit measurement based on the I-O model to derive the annual economic benefit value of Tianjin VTS.

Tianjin port is the 8th largest port globally, with a large average annual vessel flow of over 60000 vessels in the past five years. Tianjin VTS is one of the largest VTS systems in China, monitoring the only domestic compound channel, assuming the role of escorting the port economy, with the remarkable representation of operational efficiency and technological innovation capability. Tianjin VTS system has many innovative functions, such as dynamic risk assessment of water transport, intelligent projection of vessel voyage plan, 3D intelligent display, automatic alarm, Internet platform, duty simulation training system, etc. These are used to ensure the overall quality and efficiency of VTS. Therefore, this research takes Tianjin VTS as an example and uses 2016 annual data for analysis.

\section{Direct Economic Impacts Evaluation}

\subsection{Main Approaches}

Direct economic benefits refer to the economic benefits arising from the adequate performance of VTS functions by enhancing vessel traffic capacity, reducing waiting time for entry and exit the port and reducing accident losses. Since accidents are contingent and the reduction of losses is mainly based on the speculation of historical data, the accuracy is harder to grasp, so this paper only considers the saving of ship's time in port in traffic organization.

For the determination of benefits, firstly, the source of benefits should be clarified by investigating the daily work of VTS and selecting items that have a significant impact on vessels, have a large amount of operational data to analyze and can be quantified.

Secondly, collect 1-2 years of vessel traffic data before and after the above items are carried out, take the average value after sampling by vessel type, and obtain the critical parameters of the project's impact on vessel entry and exit time.

The impact parameters are determined from the historical data and combined with the operational data for the calculated year. The port access time saved for each traffic optimization project for that year can be derived.

The saved time is converted into the saved vessel operating cost, which is used as the direct economic benefit achieved by VTS through optimized traffic organization.

According to the functions of Tianjin VTS, the direct economic benefits can be divided into the following four categories.

\subsection{Evaluation}

\subsubsection{Benefits of Optimizing the Organization of Compound Channel}

Tianjin Port has the only compound channel in China, with a two-in and two-out design, realizing the diversion of large vessels and small vessels, which requests the more effective organization capacity of VTS (Figure 1). After opening the compound channel in January 2014, VTS optimized the vessel organization by system upgrade and reform to further shorten the traffic time while the vessel flow has increased significantly.

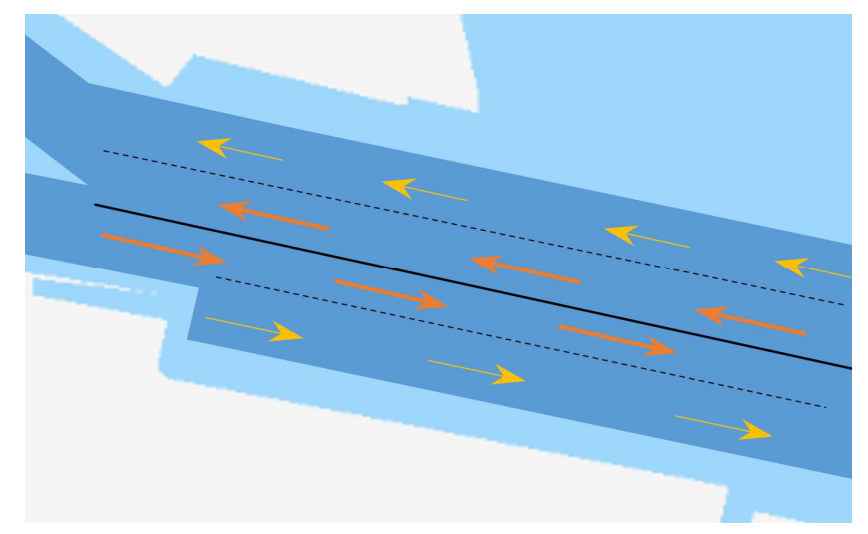

Figure 1. Compound Channel of Tianjin Port. 
(1) Navigation security under normal conditions

In calculating this benefit, a comparison of vessels' average entry and exit time before and after the commissioning of the compound channel is used.

$$
B=\Delta T \times N \times C
$$

$\Delta \mathrm{T}$ is the difference between the average entry and exit times of different types of vessels before and after the opening of the compound channel;

$\mathrm{N}$ is the number of vessels guaranteed;

$\mathrm{C}$ is the average shipping schedule cost.

According to the vessels voyage records of Tianjin VTS, the average entry and exit time per vessel were sampled and counted several times for each month in 2012 and 2013, and the average value was 99.58 minutes; in 2014 and 2015, after the opening of the compound channel, the average time per vessel was reduced to 76.33 minutes. The benefits of VTS for optimizing the organization of compound channel traffic are apparent, and the average time per vessel can be saved by 23.25 minutes, i.e. $\Delta \mathrm{T}=0.388$ hours.

The optimal organization of the VTS plays a role in improving navigational efficiency for all vessels. Still, to avoid overlap with the calculation of the benefits of the latter navigational disadvantages, only vessels up to $146 \mathrm{~m}$ in length sailing in the two small vessels channel, which benefit the most, are considered here. Because according to the channel conditions, without affecting the large vessel channel, small vessels under 146 meters in length can enter and exit the port area via the small vessel channel at the same time without waiting in line for the large vessels to complete their sailing plans. So, according to VTS statistics, $\mathrm{N}=23112$ vessels.

$\mathrm{C}$ is the average daily operating cost of vessels, primarily for small vessels. The cost is based on the vessels that have a regular operation in the port area, and the average value is taken after sampling and survey to operators, $1988 \mathrm{RMB} /$ hour.

So, the benefit generated by VTS in normal navigable conditions to shorten the time of vessels entry and exit the port through traffic organization is $\mathrm{B}=17,822,800 \mathrm{RMB}$.
(2) Clearing the backlog after particular circumstances

Vessel backlogs are a significant problem in severe weather or other particular circumstances leading to traffic control. After that, VTS will improve the regular scheme to optimize the navigation plan and guide the vessels in an orderly manner, which further shortens the vessel passage time and enhances the port productivity. In calculating this benefit, the vessels average clearing time before and after the commissioning of the compound channel is used for comparison. The shorter the clearing time, the more orderly and efficient the traffic organization, the more reasonable the arrival time and several vessels, and the lower the rate of congestion and accidents in the port.

The benefits of VTS in clearing backlog in severe weather after the compound channel opening are calculated as follows.

$$
B=\Delta T \times N \times C
$$

$\Delta \mathrm{T}$ is the difference of the average port clearance time for different types of vessels before and after the use of the compound channel;

$\mathrm{N}$ is the number of clearing vessels

$\mathrm{C}$ is the average daily operating cost of vessels;

According to the blockage clearance records of Tianjin VTS, the average detention time per vessel was 118.6 minutes in 2012 and 2013 and decreased to 83.03 minutes in 2014 and 2015. Hence, the benefits of VTS in optimizing the traffic organization of the multiple channels in bad weather are apparent, with an average saving of 35.57 minutes per vessel, or $\Delta \mathrm{T}=0.593$ hours.

In 2016, 21 critical clearances were conducted, benefiting a total of more than 1,650 vessels, with time converted in proportion to the classification of vessels in the covered area.

The average daily operating cost of vessels was determined by referring to the Drewry "Ship Operating Costs Annual Review" data, sampling the vessels by type and class, and then fitting them in proportion to the number of different vessel types in Tianjin port. The calculation results of this benefit are shown in Table 1.

\begin{tabular}{|c|c|c|c|c|c|}
\hline Representative vessel type & Container ship & Passenger Ship & Bulk/General cargo ship & Tug boat/Working ship & Tanker \\
\hline Operating Cost (RMB/day) & 145,100 & 216,100 & 119,600 & 47,700 & 113,500 \\
\hline Time saving (hour) & 211.27 & 6.56 & 340.10 & 365.51 & 55.01 \\
\hline Benefit (RMB) & $1,277,300$ & 59,100 & $1,694,800$ & 726,500 & 260,100 \\
\hline
\end{tabular}

Table 1. Benefits generated by clearing the backlog.

So, the direct economic benefit of improving port productivity under normal conditions and port evacuation by VTS optimizing the traffic organization of the compound channel in 2016 was about RMB 21,840,600.

\subsubsection{Benefits Under Adverse Navigational Conditions}

In particular situations, such as severe weather, conflicts arising from vessel encounters, large vessels occupy the main channel to turn around, etc. VTS would shorten the vessel sailing or berthing time through intelligent supervision modes like risk assessment and navigation plan projection. Thus the economic benefits are formed by the cost savings generated.
That is the primary consideration of this item.

(1) Benefits from reducing the impact of severe weather

This item includes the effects of weather factors such as poor visibility and gale force seven or higher on five types of vessels as above, mainly following the measurement proposed by Shi a (2004) [13] while adjusting some parameters according to the current situation of traffic organization.

$$
\sum_{a}=F \times n_{c} \times \Delta \% \times W
$$

$\sum_{a} \quad$ indicates the annual reduction in lost waiting time due to VTS operation; 
$F$ is the frequency of adverse conditions affecting navigation;

$n_{c}=n \times D, n$ is the average number of vessels entering and leaving the port per hour,

$n=\frac{N}{365 \times 24}, N$ is the Annual number of vessels in and out of port;

$D$ is the duration of each adverse condition;

$\Delta \%$ is the difference of vessels number waiting or decelerating between the two before and after VTS operation;
For non-tide vessels, the average waiting time is $W=\frac{D}{2}$

Five categories of vessels number in 2016, as well as the frequency and duration of 11 types of adverse weather, were counted separately, and the benefits generated by the Tianjin VTS in reducing the impact of severe weather were calculated as per the scenarios and conclusions in the above literature as shown in Table 2.

Table 2. Benefits from reducing the impact of severe weather.

\begin{tabular}{|c|c|c|c|c|c|}
\hline Representative vessel type & Container ship & Passenger Ship & Bulk/General cargo ship & Tug boat/Working ship & Tanker \\
\hline Operating Cost (RMB/day) & 145,100 & 216,100 & 119,600 & 47,700 & 113,500 \\
\hline Time saving (hour) & 45.80 & 28.40 & 290.64 & 420.03 & 20.43 \\
\hline Benefit (RMB) & 276,900 & 255,700 & $1,448,400$ & 834,800 & 96,600 \\
\hline Total (RMB) & $2,912,400$ & & & & \\
\hline
\end{tabular}

(2) Benefits arising from the reduction of vessel encounters conflicts

Due to the limitation of channel width, when large vessels sail at the main channel, the secondary channel will be closed, and some vessels have to increase the waiting time to incur delays. VTS will improve traffic efficiency by the reasonable organization. Therefore this economic benefit can be calculated as follows.

$$
B=F \times 365 \times \Delta \% \times \Delta T \times C
$$

$F$ is the frequency of encounter conflicts (vessels/day), based on a sampling of in and out vessels and the method of reference 2. We calculated that $F=91.41$ vessels in 2016 (all vessels at the time of the conflict divided by the number of days);

$\Delta \%$ is the percentage of vessels that could enter the channel earlier due to VTS organized traffic (on average 70\% according to the duty officer survey and estimates).

$\Delta T$ is the reduction in delay time per conflict vessel due to VTS organized traffic, internally assessed at 0.5 hours/vessel on average;

$C$ is the daily operating cost of the vessel. The average value is calculated as 0.4115 million yuan/hour according to the proportion of each different vessel type.

$$
B=F \times 365 \times \Delta \% \times \Delta T \times C=48,053,400 \mathrm{RMB} .
$$

(3) Benefits of reducing the adverse effects of large vessels In recent years, the size of vessels calling at Tianjin Port has gradually increased due to large-scale vessels. In addition, the location of some new terminals is close to the channel. The area available for vessels to maneuver is limited. Some large vessels have to occupy most of the whole channel when they berth and turn around at some berths, which temporarily block the traffic and affect the navigation of passing vessels. The role of VTS here is to reduce the impact of large vessels by optimizing the operation plan and reducing the number of passing vessels waiting or slowing down. The specific calculations for 2016 are as follows.

$$
B=F \times 365 \times D \times N \times \Delta T \times C
$$

$F$ is the average daily frequency of large vessels occupying the channel turnaround (vessels/day), which is $2864 / 365=7.85$ vessels per day;

$D$ is the average duration of each time (hour);

$N$ is the average number of vessels in and out of the port per hour, which is $60710 / 365 / 24=6.9303$ vessels per day;

$\Delta T$ is the average reduction in delay time per vessel (hours);

$$
B=9,805,400 \text { RMB. }
$$

\subsubsection{Benefit of Smart Services}

Tianjin VTS changes the traditional way of releasing traffic information by using VHF, building a multi-level network information platform, and actively exploring the new VTS information service mode by integrating online government services and mobile intelligent terminal (APP). The AIS intelligent application technology is deeply explored to automatically push and distribute relevant navigation plans, traffic control, navigation warnings, and other VTS information to achieve the information self-service assessment for vessels and to realize intelligent real-time interaction of ship-shore.

The Tianjin VTS traffic information service platform is one of the applications that greatly facilitates small vessels' production arrangement. It has shortened the long and traditional process of declaration through agents. Now it only needs to declare online 4 hours in advance, and the process of declaration to approval saves 2-6 hours.

The relevant benefits are calculated as follows:

$$
B=N \times \Delta T \times C=12,942,798 \mathrm{RMB}
$$

$N$ is the number of unplanned vessels declared through the information service platform, which is the number of vessels approved by the platform for the year $8589 \mathrm{x}$ the proportion of unplanned vessels $37.9 \%$;

$\Delta T$ is the approval time limit savings, take the minimum value of 2 hours;

$C$ is the daily operating cost of the vessel. Because most of them are small vessels, the cost is taken as $1988 \mathrm{RMB} /$ hour for harbour craft. 


\subsubsection{Reducing the Cost of Water-related Projects}

VTS has made every effort to ensure the safety of construction operations of several major projects by understanding the characteristics of the projects in advance, customizing targeted solutions and managing the construction throughout the process. Company $\mathrm{H}$ is a representative owner in the area with the most construction works. Therefore, the benefit mainly considers the company's channel dredging and water maintenance operations.

The construction projects usually involve many vessels and extensive waterborne. The operations often conflict with the regular production schedule of the port, affect the navigation and berthing of vessels, and are also subject to the constraints of fog, wind and other severe weather.

VTS's customized services include assistance in making plans in advance, guidance and information interaction during construction, and post-construction evaluation. The economic benefits mainly come from shortening project time and saving project costs by optimizing plans and processes. The calculation is as follows.

$$
B=\sum_{i=1}^{n} \Delta T_{i} \times\left(C_{i} \div 24\right)
$$

$\Delta T_{i}$ is the difference between the actual operating time and the planned available time for each project;
$\mathrm{C}_{\mathrm{i}}$ is the average daily construction cost per project;

After investigation and statistics, the planned operating days of the waterside project in 2016 were 247 days and the actual operating days were 115 days. The average time utilization rate of the project was $65 \%$, and the time utilization rate considering only weather, breakdown and regular traffic restrictions was $50 \%$, so $15 \%$ of the time utilization rate enhancement was counted as VTS regulatory benefit. The construction cost mainly considers the average daily cost of chartering vessels of 300,000 RMB. That is $\mathrm{B}=15 \% \times 115 \times 300000=5,175,000 \mathrm{RMB}$.

\subsection{Result}

Tianjin VTS applies the innovative integrated management mode of intelligent control for the compound channel, improving service level and creating significant social and economic benefits. Through multi-dimensional indicators such as vessel tracking rate, water hazard rate, and information service provision capability, the critical role played by VTS in improving navigation efficiency, guaranteeing water safety, and ensuring port productivity can be reflected. The direct economic benefits that can be further quantified and applied to monetized measurement are shown in Table 3.

Table 3. Summary of direct economic benefits of Tianjin VTS.

\begin{tabular}{lll}
\hline Direct economic benefits & & Amount (Million Chinese Yuan) \\
\hline $\begin{array}{l}\text { Optimizing the organization of compound } \\
\text { channel }\end{array}$ & Navigation security under normal conditions & 17.82 \\
& Clearing the backlog after particular circumstances & 4.02 \\
In adverse navigational conditions & Reducing the impact of severe weather & 2.91 \\
& Reduction of vessel encounters conflicts & 48.05 \\
Smart services & Reducing the adverse effects of large vessels & 9.81 \\
Reducing the cost of water-related projects & & 12.94 \\
Total & & 5.18 \\
\hline
\end{tabular}

\section{Indirect Economic Impacts Evaluation}

The indirect economic benefits of VTS refer to the economic pull effect of VTS through promoting the development of the water transportation industry and thus further promoting or driving the development of other related sectors, mainly including backward ripple effect and forward ripple effect. The economic benefits are mainly assessed by the input-output method (I-O model).

The connection of VTS consuming products of other sectors is called the backward ripple effect, such as purchasing patrol vessels. The connection between VTS and shipbuilding is backward. VTS demand and improve the output value and added value of shipbuilding is the backward ripple effect of VTS to shipbuilding.

VTS service as an intermediate product of other sectors is called forward ripple effect, such as the safe navigation of vessels need VTS to provide information services. Therefore, part of the revenue generated by the shipping company should come from VTS, marine and shipping company's link is called forward link, the corresponding effect is the forward ripple effect.

\subsection{Methodology}

\subsubsection{Backward Ripple Effect}

As mentioned above, the backward ripple effect of VTS is calculated through the backward ripple effect of the water transportation industry. The value of complete inputs required per unit of output and services of products from other intermediate sectors for the water transportation industry $j$ can be obtained from the $C_{j}=\left(c_{\mathrm{ij}}\right)_{n \times 1}$ through the $j$ column of the complete consumption coefficient matrix (Leontief's inverse-Identity matrix), where $j$ is the water transportation industry and $i=1,2, \ldots$, $\mathrm{n}$ represents the remaining sectors. The contribution of the water transportation industry is the total output and GDP generated by the water transportation industry when acting alone to each of the remaining sectors and the entire economy.

Water transportation industry backward ripple and GDP contribution:

$$
E_{b i}=C_{j} \cdot \Delta X_{i j}
$$

$j$ is the water transportation industry, $i=1,2, \ldots$ for other sectors; 
$\Delta \mathrm{X}_{\mathrm{ij}}$ is the total output of the water transportation industry; $C_{j=}\left(c_{i j}\right)_{n \times 1}$ is the output of the water transportation industry $\mathrm{j}$ per unit of product and service requires the total value of inputs from other intermediate sector products.

\subsubsection{Forward Ripple Effect}

The water transportation industry is an essential intermediate input. Its development, on the one hand, needs the input of other sectors. On the other hand, it is invested in other sectors to promote its development, resulting in a forward ripple effect. Tianjin is a coastal city, the forward ripple effect of the water transportation industry on the economic development of Tianjin is also more prominent.

When the water transportation industry increases output value $\Delta \mathrm{x}$, some products and services will be distributed as intermediate inputs. Assuming these sectors balanced develop, each sector requires the same proportion of intermediate inputs to the water transportation industry. The value of water transportation industry products and services required by sector $\mathrm{j}$ is as follows.

$$
u_{\mathrm{ij}}=\left\{\begin{array}{c}
h_{\mathrm{ij}} X_{i} \mathrm{j}=1,2, \ldots, \mathrm{n}(j \neq i) \\
0 \mathrm{j}=\mathrm{i}
\end{array}\right.
$$

$X_{i}$ is the total output of the water transportation industry in the sectoral distribution of intermediate inputs;

$h_{\mathrm{ij}}$ is the element corresponding to column $\mathrm{j}$ and row $\mathrm{i}$ of the distribution factor Figure.

The primary forward ripple output or total output of the intermediate inputs allocated in sector $\mathrm{j}$ to the other sectors is

$$
\Delta x_{\mathrm{ij}}=\left\{\begin{array}{c}
u_{\mathrm{ij}} / a_{\mathrm{ij}} a_{\mathrm{ij}} \neq 0 \\
0 a_{\mathrm{ij}}=0
\end{array}\right.
$$

$a_{\mathrm{ij}}$ is the coefficient of intermediate inputs required in sector $i$ for a unit of output in sector $j$.

It is assumed that equal amounts of intermediate inputs in a sector can produce equal proportions of total product and value-added, without considering the changes in input-output ratios caused by short-term factors in the sector and expressed in direct consumption coefficients.

The total output of the water transportation industry and its corresponding distribution coefficient $h_{\mathrm{ij}}$ are substituted into Equations 8 and 9. The intermediate inputs required for each sector of the water transport industry can be obtained separately, as well as the water transport industry forward ripple value-added and vector $\Delta X_{j}$ of output value that each sector can generate.

When the sectors expand production, besides increasing the water transport industry as an intermediate input, there is a proportional increase in the products of other sectors as intermediate inputs, that is $C \Delta X_{j}^{T}$.

Thus, the forward ripple effect in the water transportation industry is

$$
E_{\mathrm{fl}}=z \cdot \Delta X_{j}^{T}+z \cdot C \cdot \Delta X_{j}^{T}
$$

$\mathrm{z}$ is the vector of GDP value-added coefficients within the region;

$z_{j}=\frac{G_{j}}{X_{j}}$ is the GDP $\left(G_{j}\right)$ that can be created by increasing one unit of gross output $\left(X_{j}\right)$ in sector $\mathrm{j}$;

$\Delta X_{j}^{T}$ is the transpose of the primary forward ripple production value-added and vectors for each sector, and $\mathrm{C}$ is the matrix of fully consumed coefficients.

\subsection{Data}

Due to the limitation of data collection, the 2012 version of "Tianjin Input-Output Table" was used. Seven sectors, including agriculture, industry, construction, comprehensive transportation (excluding water transportation), modern service industry and other industries, which are most closely related to the water transportation industry, were sorted out. The adjusted input-output adjustment table of the seven sectors is shown in Table 4.

Table 4. Simplified structure of Tianjin I.O. table in 2012

Currency unit: Million Chinese Yuan

\begin{tabular}{lllllll}
\hline & Agriculture & Industry & Construction & $\begin{array}{l}\text { Integrated } \\
\text { transport }\end{array}$ & $\begin{array}{l}\text { Water } \\
\text { transport }\end{array}$ & $\begin{array}{l}\text { Modern } \\
\text { services }\end{array}$ \\
\hline Agriculture & 3919 & 61122 & 308 & 140 & 29 & 15823 \\
Industry & 12855 & 1578440 & 199899 & 38052 & 23538 & 116458 \\
Construction & 65 & 1116 & 2217 & 1102 & 30 & 3669 \\
Integrated transport & 1352 & 50226 & 7301 & 67639 & 24306 & 16347 \\
Water transport & 134 & 9398 & 494 & 5971 & 13890 & 1287 \\
Modern services & 2058 & 163729 & 41050 & 23358 & 14784 & 162217 \\
Other industries & 17 & 667 & 168 & 309 & 204 & 3966 \\
Intermediate use & 20403 & 1864702 & 251439 & 136573 & 76783 & 319769 \\
Labor Compensation & 16217 & 214802 & 25871 & 26226 & 7937 & 141959 \\
Depreciation of fixed assets & 0 & 94474 & 10846 & 10333 & 1199 & 96318 \\
Net tax on production & 943 & 84566 & 5674 & 7993 & 2732 & 43428 \\
Operating Surplus & 0 & 218462 & 11685 & 12613 & -684 & 178622 \\
Value added & 17160 & 612306 & 54076 & 57167 & 11185 & 460327 \\
Total input & 37563 & 2477008 & 305515 & 193741 & 87968 & 780097 \\
\hline
\end{tabular}




\begin{tabular}{lllllll}
\hline & $\begin{array}{l}\text { Other } \\
\text { industries }\end{array}$ & $\begin{array}{l}\text { Intermediate } \\
\text { use }\end{array}$ & $\begin{array}{l}\text { Final } \\
\text { consumption }\end{array}$ & Accumulation & Net imports & Total output \\
\hline Agriculture & 70 & 81414 & 26271 & 858 & 70981 & 37563 \\
Industry & 37788 & 2007032 & 133975 & 191629 & -144371 & 2477008 \\
Construction & 3176 & 11377 & 5235 & 646238 & 357336 & 305515 \\
Integrated transport & 3992 & 171167 & 7968 & 2634 & -11970 & 193741 \\
Water transport & 47 & 31223 & 465 & 333 & -55946 & 87968 \\
Modern services & 22247 & 429446 & 134202 & 143149 & -73299 & 780097 \\
Other industries & 5262 & 10595 & 179820 & 0 & 40665 & 149750 \\
Intermediate use & 72584 & 2742256 & 487938 & 984844 & 183395 & 403164416 \\
Labor Compensation & 71023 & 504037 & & & & \\
Depreciation of fixed assets & 642 & 213814 & & & & \\
Net tax on production & 5338 & 150675 & & & & \\
Operating Surplus & 161 & 420860 & & & & \\
Value added & 77165 & 1289388 & & & & \\
Total input & 149750 & 4031644 & & & & \\
\hline
\end{tabular}

\subsection{Result}

\subsubsection{VTS Backward Ripple Effect}

According to the Tianjin I.O. table, the backward ripple correlation coefficient of the water transportation industry to other industries can be calculated. The correlation coefficient is applied to VTS as a component of the water transportation industry. The corresponding backward ripple effect can be calculated by combining the total direct benefits of VTS.

According to Equation 7, $j$ is the water transportation industry, $i=1,2, \ldots \mathrm{n}$ for other sectors, $\Delta \mathrm{X}_{\mathrm{ij}}$ is the total output of the water transportation industry, taking the value of direct benefits of $100,729,598 \mathrm{RMB}, \mathrm{C}_{\mathrm{j}=}\left(\mathrm{c}_{\mathrm{ij}}\right)_{\mathrm{n} \times 1}$ is the output of the water transportation industry $j$ per unit of product and service requires the total value of inputs from other intermediate sector products.

The Simplified structure of the Tianjin I.O. table (Table 4) was used to calculate the complete consumption coefficient matrix, as shown in Table 5 .

Table 5. Complete consumption coefficient matrix.

\begin{tabular}{|c|c|c|c|c|c|c|c|}
\hline & Agriculture & Industry & Construction & $\begin{array}{l}\text { Integrated } \\
\text { transportation }\end{array}$ & $\begin{array}{l}\text { Water } \\
\text { transportation }\end{array}$ & $\begin{array}{l}\text { Modern } \\
\text { services }\end{array}$ & $\begin{array}{l}\text { Other } \\
\text { industries }\end{array}$ \\
\hline Agriculture & 0.156014817 & 0.090396434 & 0.068395826 & 0.040703735 & 0.052329011 & 0.048454977 & 0.034312813 \\
\hline Industry & 1.259571442 & 2.044295926 & 2.127002895 & 1.132740876 & 1.474379624 & 0.657989603 & 0.976647537 \\
\hline Construction & 0.004297969 & 0.003617315 & 0.010990724 & 0.011670349 & 0.006910199 & 0.00727667 & 0.024616353 \\
\hline $\begin{array}{l}\text { Integrated } \\
\text { transportation }\end{array}$ & 0.116602799 & 0.117250368 & 0.126759187 & 0.613767255 & 0.581145823 & 0.070316206 & 0.089119846 \\
\hline $\begin{array}{l}\text { Water } \\
\text { transportation }\end{array}$ & 0.015263236 & 0.018954725 & 0.017196979 & 0.065069015 & 0.216589193 & 0.008378016 & 0.008831739 \\
\hline $\begin{array}{l}\text { Modern } \\
\text { services }\end{array}$ & 0.207220238 & 0.283286987 & 0.377331194 & 0.359726919 & 0.475729127 & 0.335904122 & 0.298276107 \\
\hline Other industries & 0.002228985 & 0.002628599 & 0.003443844 & 0.00506795 & 0.006834814 & 0.007387133 & 0.03846471 \\
\hline
\end{tabular}

So, $E_{b i}=2.81391779 \times 100,729,598=283,444,808$ RMB.

\subsubsection{VTS Forward Ripple Effect}

According to equation $10, \mathrm{Z}$ is the coefficient of GDP, the economic data of the Tianjin Statistical Yearbook in 2016 were selected, and the calculation results are shown in Table 6.

Table 6. GDP value-added coefficient of Tianjin.

\begin{tabular}{ll}
\hline Agriculture & $\mathbf{0 . 4 5 6 8 3 2 5 2 1}$ \\
\hline Industry & 0.247195813 \\
Construction & 0.176999529 \\
Integrated transportation & 0.295070771 \\
Water transportation & 0.127147883 \\
Modern services & 0.590090298 \\
Other industries & 0.515294456 \\
\hline
\end{tabular}

$\Delta \mathrm{X}_{\mathrm{j}}^{\mathrm{T}}$ is each sector of the primary forward ripple and value-added output and vector transpose, according to equations 8 and 9, sector $i$ requires VTS products and services as listed in Table 7.
Table 7. The value of products and services sectors need from the water transportation industry.

\begin{tabular}{ll}
\hline & Water transportation \\
\hline Agriculture & 4983.9409 \\
Industry & 328654.8489 \\
Construction & 40536.4046 \\
Integrated transportation & 25706.0125 \\
Water transportation & 0 \\
Modern services & 103505.0401 \\
Other industries & 19869.2462 \\
\hline
\end{tabular}

So, $E_{f l}=1,487,733,711 \mathrm{RMB}$.

According to the industry linkage theory, VTS, as an integral part of the water transportation industry, has a direct economic contribution to the water transportation industry and further promotes the development of other sectors and generates an indirect economic pull. It enhances the 
competitiveness of the regional economy and provides a solid guarantee for the development of port services and the shipping economy. Accordingly, the indirect economic benefits of VTS in Tianjin in 2016 calculated by the input-output method are shown in Table 8.

Table 8. Indirect benefits of Tianjin VTS in 2016.

\begin{tabular}{ll}
\hline Category of benefits & Amount (Million Chinese Yuan) \\
\hline Backward ripple effect & 283.44 \\
Forward ripple effect & 1487.73 \\
Total & 1771.18 \\
\hline
\end{tabular}

\section{Conclusion}

Research on the economic evaluation of VTS has been developing. This paper proposes dividing such benefits into direct and indirect economic benefits, argues that VTS generates direct economic benefits by shortening the time of vessels in port and creating added value for the port economy through traffic organization optimization. Additionally, it contributes to the upstream industry by pulling through the consumption of raw materials and fuel. It also contributes to the downstream industry by providing transportation consulting and waterway management services, creating indirect economic benefits.

Based on the typical operation of Tianjin VTS and a large volume of business data, a calculation model of direct benefits was established, resulting in 100.73 million RMB (about 14.52 million USD). Based on the Input-Output model, the indirect benefits are calculated to be 1771.18 million RMB (about 255.32 million USD).

Besides focusing on the economic contribution value, more factors can be further identified from the management system, environment and staff to improve the socio-economic benefit evaluation of VTS. Qualitative and quantitative evaluation indicators can be increased to establish a more comprehensive evaluation system.

At present, VTS continues to carry out the intelligent transformation, with increasingly powerful information collection and optimized operation capabilities and has accumulated a large amount of business data in practice. Further theoretical research can focus on deep mining and analysis of business data by using big data technology to realize evaluation based on total data and make up for the current shortage of mainly using sampling analysis. In the big data era, it is significant to exploit the application value of ship traffic management data fully. It will lead to more scientific and reasonable results, which is what this project will do.

\section{Acknowledgements}

The work presented in this paper is financially supported by Tianjin Maritime Administration, Fujian Education Department (Grant number JAT160272) and Jimei University (Grant number ZC2016020).

\section{References}

[1] M. Wm. Hans, (1976). COST-BENEFIT ANALYSIS FOR VESSEL TRAFFIC SYSTEMS. Proceedings of an international symposium, p. 11-15.

[2] Tian H. W., (2000) Cost-Benefit Analysis of Vessel Traffic Management System, Dalian Maritime University, p. 64.

[3] Liu, R. J., Shao, F. M. \& Liu, X. O. (2001). Evaluation issues of investment and benefit of VTS system. Proceedings of the 2001 Annual Academic Exchange Conference of the China Navigation Society, p. 129-132.

[4] Shao, C. F., (2002) Vessel Traffic Management System Benefit Evaluation and Hazard Assessment, Dalian Maritime University, p. 72.

[5] George Bushell, Dr Tony Quon, Peter Everson. (1992). Assessing the benefits and costs of VTS: A Canadian example with emphasis on risk assessment and environment protection, Proceedings of the seventh international symposium on vessel traffic services, Canada, June.

[6] IALA, Vessel Traffic Services Manual, Ed. 6. June 2016.

[7] Xie Xie, Gao Yu. (2010). Methodology for measuring the social contribution of maritime economy. China Maritime, 05, $52-55$.

[8] Lu, H. (2016). Study on the effectiveness evaluation of Qingdao VTS. Dalian Maritime University.

[9] Li Huawen. (2010). Economic and social impact evaluation of ship traffic management system. Dalian Maritime University.

[10] Cao Desheng. (2018). Introduction to performance assessment of ship traffic management system. People's Traffic Publisher.

[11] Lee, G., Kim, S. Y., \& Lee, M. K. (2015). Economic evaluation of vessel traffic service (VTS): A contingent valuation study. Marine Policy, 61, 149-154.

[12] Mou, J. M., Zhou, C., Du, Y., \& Tang, W. M. (2015). Evaluate VTS benefits: A case study of Zhoushan Port. International Journal of e-Navigation and Maritime Economy, 3, 22-31.

[13] Shi, G., Fang, X. (2004). Research on Evaluation of Benefit and Risk Assessment of Port VTS. International Symposium on Vessel Traffic Services, ISVTS2004, Hong Kong, pp. 111-119. 